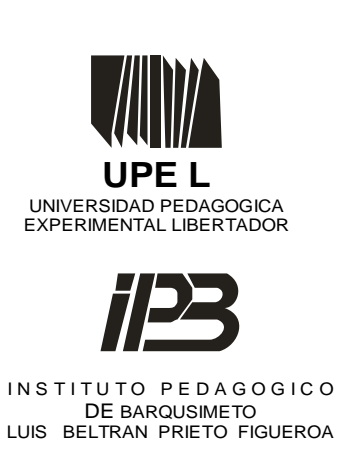

DE BARQUSIMETO
LUIS BELTRAN PRIETO FIGUEROA

\section{REVISTA}

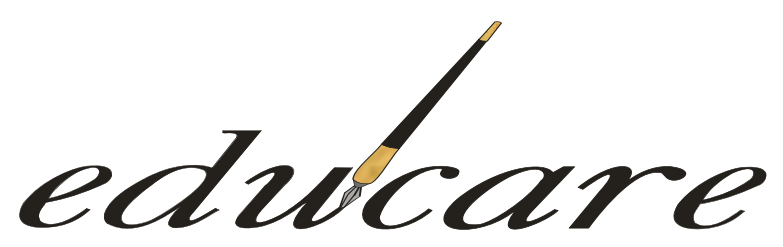

Órgano Divulgativo de la Subdirección de Investigación y Postgrado del Instituto Pedagógico de Barquisimeto "Luis Beltrán Prieto Figueroa"

BARQUISIMETO - EDO. LARA - VENEZUELA

\title{
NUEVA ETAPA
}

FORMATO ELECTRÒNICO

DEPOSITO LEGAL: ppi201002LA3674

Volumen 17 № 2

ISSN: 2244-7296

\section{MANUAL DIDÁCTICO PARA EL USO DEL DICCIONARIO BILINGÜE INGLÉS-ESPAÑOL/ ESPAÑOL-INGLÉS \\ ENGLISH-SPANISH / SPANISH-ENGLISH DICTIONARY DIDACTIC MANUAL}

\author{
Loisineth Suárez \\ U.E. "Rafael Antonio Mujica" \\ Gerardo Corbo \\ Universidad Pedagógica Experimental Libertador \\ Instituto Pedagógico de Barquisimeto. \\ Venezuela
}




\section{MANUAL DIDÁCTICO PARA EL USO DEL DICCIONARIO BILINGÜE INGLÉS-ESPAÑOL / ESPAÑOL-INGLÉS}

ENGLISH-SPANISH / SPANISH-ENGLISH DICTIONARY DIDACTIC MANUAL

\section{INVESTIGACION}

Recibido:06-05-13

\author{
Loisineth Suárez Pérez ${ }^{*}$ \\ U.E. "Rafael Antonio Mujica" \\ Gerardo Corbo** \\ UPEL-IPB \\ Venezuela
}

\begin{abstract}
RESUMEN
En el aprendizaje de una lengua extranjera el diccionario bilingüe es una herramienta valiosa; más, si se sabe emplear. El presente estudio tiene como objetivo principal reportar los hallazgos de la Fase de Diagnóstico de un Trabajo de Investigación bajo la modalidad de un proyecto especial. Este proyecto tuvo como propósito proponer un manual para el uso del diccionario bilingüe español-inglés / inglés- español. El trabajo se enmarca en la línea de investigación Metodología de la Enseñanza-Aprendizaje del Inglés bajo un enfoque cuantitativo y se ubica dentro de la modalidad de Proyecto Especial, apoyado en un estudio de campo. La población está conformada por 40 estudiantes de cuarto año a quienes se les aplicó una prueba de habilidad sobre el uso del diccionario. Los resultados arrojaron que los participantes presentan muchas dificultades al momento de localizar el adecuado equivalente en español de palabras con cambios morfológicos, polisémicas y de frases lexicales, lo que refleja el poco dominio de los estudiantes del diccionario bilingüe y que justifica la propuesta de un manual para guiar el uso del mismo.
\end{abstract}

Descriptores: Diccionario Bilingüe inglés-español, uso del diccionario, manual, enseñanza del inglés
Aceptado:14-07-13

\begin{abstract}
In the process of learning a foreign language the bilingual dictionary is a valuable tool, even more, if it is known how to use it. The present study has as its main objective the design of a teaching manual for the use of the bilingual dictionary EnglishSpanish/Spanish-English with the purpose of providing the necessary tools to benefit from the dictionary mentioned before. The study is framed in the line of research English learning-teaching methodology, on a quantitative approach within the modality of Special Project, supported in a field study. The population consists of 40 fourth-year students who were applied a test of skill on the use of the bilingual dictionary. The results showed that the participants have many difficulties locating the appropriate Spanish equivalent of words with morphological changes, with multiple meanings and with lexical phrases which justifies the design of the proposal.
\end{abstract}

Keywords: Bilingual Dictionary English-Spanish, dictionary use, manual, teaching English

\footnotetext{
*Profa. Loisineth Suarez Pérez. Egresada de la UPEL-IPB con el título de profesora en la especialidad de inglés. Magister en Enseñanza del Inglés como Lengua Extranjera. Docente de la UE "Rafael Antonio Mujica” Loisinethsuarezp@ @otmail.com

**Prof. Gerardo Corbo. Docente investigador adscrito al Departamento de Idiomas. UPEL-IPB. Magister en Enseñanza del Inglés como Lengua Extranjera. PEII 2012, Nivel A. gerardocorbo@ hotmail.com
} 


\section{INTRODUCCIÓN}

En el aprendizaje de una lengua, ya sea como segunda lengua o como lengua extranjera, el diccionario bilingüe es una herramienta valiosa, Benedikt (2005) plantea que "el diccionario bilingüe es uno de los diferentes tipos de diccionarios que nos ayudan a aprender y utilizar lenguas extranjeras". Así mismo, este autor (ob.cit.) expresa que "incluso a la hora de comprender un texto hay casos donde la consulta de un diccionario bilingüe resulta más útil”. De igual forma, Fernández (1999) expresa que el diccionario es una herramienta obligatoria, aunque los aprendices deben tratar de inferir por contexto el máximo posible de vocabulario.

Por otra parte, el uso adecuado del diccionario bilingüe contribuye al conocimiento de vocabulario, tal como lo afirman Luppescu y Day (1993) en su investigación sobre la contribución del diccionario bilingüe en el aprendizaje de vocabulario en inglés, Reading Dictionaries and Vocabulary Learning. Otros autores como: Enecoiz (2000), Cote y Tejedor (1998), Llorente y Fitzhugh (s.f), plantean en sus estudios la importancia de la enseñanza del uso correcto del diccionario bilingüe.

Con el objetivo de ayudar a los estudiantes a hacer un mejor uso de los diccionarios bilingües inglés- español/español inglés, el presente trabajo muestra el diseño de un manual didáctico. El estudio se enfocó en un grupo de estudiantes cursantes de cuarto año de la U. E. "Rafael Antonio Mujica" ubicada en el caserío Guararute del Municipio Arístides Bastidas, Yaracuy. Entre sus aportes más importante, aparte del manual, se encuentra la revisión realizada por la autora sobre las obras lexicográficas que emplean los sujetos de investigación, la cual sirvió de soporte teórico sobre la macro y microestructura de los diccionarios que usan la población en estudio; información que posteriormente fue empleada en la elaboración del manual.

La propuesta del manual surgió a partir de un diagnóstico que se realizó sobre los estudiantes objeto de estudio, en el cual se evidenció las deficiencias que presentan en el empleo adecuado de los diccionarios bilingües inglés- español. Por ello se procedió al diseño del manual con la finalidad de transformar la realidad evidenciada. A través de ella se procura aportar las herramientas necesarias a los educandos para que busquen, de una manera eficiente, el equivalente de las palabras en español según el contexto en el cual se encuentre, y de esta manera puedan obtener un máximo provecho del mencionado recurso. 
Con el uso correcto y eficiente de este instrumento se estima que el estudiante pueda construir sus propios conocimientos de forma autodidacta. Esta propuesta se considera relevante puesto que a través de ella se persigue transformar la realidad evidenciada y podrá ser empleada con cualquier otra población estudiantil que presente las mismas características cognitivas y socioculturales de los sujetos que forman el campo de estudio.

\section{FUNDAMENTACIÓN REÓRICA}

Como base teórica para respaldar la propuesta que se plantea, se tomó los conocimientos lexicográficos de Francisco J. Pérez (2005) y Elena Bajo Pérez (2000) con apoyo de una revisión realizada por la autora sobre las obras lexicográfica empleadas por los estudiantes objetos de estudio. También se consideró la teoría de Jeremy Harmer sobre el vocabulario y la categoría gramatical de las palabras, así como los fundamentos teóricos para la elaboración de manuales didácticos del Instituto Técnico de Capacitación y Productividad (INTECAP).

\section{Lexicografía}

Hacer investigación sobre el empleo de diccionarios, es trabajar sobre lexicografía. La lexicografía es, según Bajo (2000), "la disciplina que estudia la planificación y elaboración de compilaciones léxicas", es decir, ésta se encarga de la elaboración de diccionarios y glosarios entre otros. Aunque no se pretende elaborar un diccionario, es necesario familiarizarse con todo lo referente a dicho recurso para poder idear o agrupar actividades didácticas sobre el uso del mismo. Entre los aspectos a estudiar se encuentran su definición, uso o función, tipos y cómo está estructurado (macroestructura y microestructura). Todos estos aspectos son abordados según los fundamentos teóricos de Pérez (2005) y Bajo (2000).

\section{El Diccionario}

De acuerdo con Bajo (2000), el diccionario "es un catálogo o colección de unidades léxicas definidas o explicadas de algún modo, que se disponen en un orden determinado". Pérez (2005), por su parte, lo define como "un género de obra que ordena alfabéticamente el universo léxico de una lengua o de una disciplina..." en palabras más sencillas, es un libro que compila un determinado número de unidades léxicas (palabras), 
de una o más lenguas o áreas de conocimiento, con el objeto de definirlas, explicarlas o exponer su traducción en otra lengua, según el propósito con que haya sido elaborado.

Con respecto a su función, Pérez (ob.cit) expresa que no es más que un instrumento auxiliar del estudio o de la investigación, que busca ser un material referencial en el cual se puede localizar el origen de las palabras, su categoría gramatical, explicación semántica o pragmática, usos apropiados, contextos posibles en donde se desempeñan, voces o conceptos relacionados y otras acepciones. El uso en sí va a depender del objetivo con que haya sido creado y del usuario que lo utilice.

Hay diferentes tipos de diccionarios u obras lexicográficas. La primera categorización se hace de acuerdo al usuario. Según Bajo (ob. cit) para los usuarios con conocimiento y dominio del idioma les corresponden los diccionarios generales (definitorios, de uso...) y para aquellos en fase de aprendizaje les corresponden los escolares, ya sean bilingües o monolingües. Por lo general, existen una gran variedad de tipos de diccionarios: generales, regionales, históricos, dialectales, bilingües, técnicos (especializados un una sola área de conocimiento), de sinónimos y antónimo, etc. El que se empleará en este estudio es el bilingüe (inglés-español/español-inglés) debido a las características de los objetos de estudio de esta investigación.

En lo concerniente a su estructura, Pérez (ob. cit) y Bajo (ob. cit) concuerdan en que los diccionarios los constituyen una macroestructura y una microestructura.

\section{Macroestructura}

Según Pérez (ob. cit) es la estructura general del diccionario. Ésta establece los criterios para el ordenamiento de los artículos y para el diseño de las pautas tipográficas. Los elementos que conforman los criterios son los siguientes:

a) El cuerpo descriptivo: conjunto que reúne todas las voces que se consideran materia léxica para ser explicada.

b) La introducción metodológica, en donde el autor o autores del diccionario exponen los criterios de la investigación lexicográfica.

c) Las normas para el uso del diccionario, que es un apartado "obligado" en donde se explica las pautas de uso destinadas a los usuarios.

d) La tabla de abreviaturas y símbolos.

e) Los apéndices sobre algunos tópicos desincorporados al corpus general de la obra. 


\section{Microestructura}

De acuerdo con Pérez (ob. cit) la microestructura o estructura de los artículos, se ocupa de establecer los tópicos explicativos que funcionan sistemáticamente para cada uno de los artículos que conforman el corpus del diccionario. Otras denominaciones posibles según Bajo (ob. cit) son ítem, entrada y lema. Esta última se reserva para el encabezamiento de cada artículo. Los elementos microestructurales según Pérez (ob. cit) son los siguientes:

a) El lema o cabeza del artículo.

b) La marca gramatical que señala la categoría gramatical de la voz.

c) Las marcas que señalan la categoría de uso estilísticos (coloquial, popular, restringido...) en que funcionan las voces.

d) La definición que consiste en una explicación semántica.

e) Los contextos, que son fragmentos lingüísticos que indican los usos concretos de las voces.

f) Las observaciones, que son comentarios gramaticales o enciclopédicos sobre la voz lema.

Con referencia a la microestructura de los diccionarios escolares bilingües, en la definición, no se busca dar una explicación semántica sino una equivalencia semántica en otra legua. Pérez (ob. cit) expresa que esto lleva a entender la diferencia tipológica fundamental entre los diccionarios que describen una lengua y los que describen varias. En este sentido, los diccionarios bilingües y poliglotas, según el autor en cuestión, "conciben su cuerpo descriptivo como un haz de equivalencias (semánticas o pragmáticas), en donde se enfrentan, una lengua de partida con otra u otras de llegada." (p25)

Un aspecto importante dentro de la sistematización de los elementos microestructurales, es la presencia de ejemplos. Según Pérez (ob.cit) los ejemplos, "se aducen esencialmente para facilitar el uso apropiado de la palabra: deben aclarar la definición o cualquiera de las otras informaciones contenidas en el artículo" (p45). De igual manera, el mismo autor expone que aparte de que el ejemplo ayuda a comprender mejor la definición, permite ver con qué otras palabras se combinan preferentemente y de qué modo lo hace. Efectivamente los ejemplos son de mucha ayuda para los estudiantes, en 
especial para los que intentan aprender una lengua extranjera como el inglés. El hecho de ver las palabras en contextos concretos, permite al aprendiz emplearlas correctamente. Bajo (ob.cit), dentro de sus estudios lexicográficos, expresa que:

El valor de un diccionario como obra de consulta depende de la calidad de su contenido, lo cual no impide que las características físicas del mismo faciliten o dificulten su uso...Dado que los diccionarios son como son..., la solución consiste en aprender a usarlos. (p52-53).

Éste es, precisamente, el objetivo principal de esta investigación. Que los estudiantes de cuarto año de la U.E. "Rafael Antonio Mujica" aprendan a usar dichos instrumentos correctamente.

\section{Resumen de la Revisión de los Diccionarios que Emplean los Estudiantes que Conforman el Campo de Estudio. \\ (Oxford, Norma, Larousse: básico y pocket, Chicago universal, Chicago Tower, Chicago City, New Grand Chicago Universal y Universidad de Miami)}

En correlación con los planteamientos lexicográficos de Bajo y Pérez (ob.cit), y en búsqueda del logro del objetivo propuesto, se realizó una revisión documental sobre los diccionarios que emplean los estudiantes que conforman el campo de estudio, con el objeto de profundizar los conocimientos sobre los diccionarios bilingües inglés-español, específicamente sobre los que ellos utilizan.

Para su realización se procedió a describir cada uno de los diccionarios dentro de su estructura (macro y micro) con la finalidad de conocer sus similitudes y diferencias, fortalezas y debilidades. No con la intención de discriminar uno con otros, sino con el propósito de tener un norte, de modo que al momento de delinear las actividades prácticas del manual sobre el uso del diccionario, éstas correspondan con los diccionarios que ellos manejan.

En lo que se refiere a la macroestructura, según dicha revisión, se obtuvo que:

a) El diccionario bilingüe inglés-español lo constituyen dos estructuras, una macro que se enfoca en la estructura general del diccionario y una micro que se centra en la estructura interna de cada uno de los artículos, tal como lo señalan Bajo y Pérez en sus escritos.

b) El diccionario bilingüe inglés-español es frecuentemente bidireccional debido a que puede emplearse para realizar traducciones a ambos idiomas y por ello se divide en dos segmentos inglés-español/español inglés, que generalmente se organizan de 
forma alfabética. En las hojas preliminares muestra la introducción o el prólogo, índice, lista de abreviaturas e información adicional de tipo gramatical y fonética. Algunos diccionarios presentan instrucciones de uso.

c) La mayoría de estos recursos facilita algunos apartados (apéndices) al inicio, en el centro o al final, en las cuales proporciona listas de verbos, números, ilustraciones u otra clase de información complementaria. Por lo general, cada página izquierda del corpus, en la parte superior izquierda presenta un vocablo (palabra guía, de entrada) el cual indica que es el primer término con que comienza la página. Asimismo, la página derecha muestra un vocablo en la parte superior derecha (palabra de salida) que indica que es el último de esa página.

Como se puede evidenciar, los elementos macro estructurales (introducción, tabla de abreviaturas, instrucciones...) tienen correspondencia con los criterios expresados por Pérez (ob.cit), descritos en párrafos anteriores, sobre la macroestructura de los diccionarios.

a) Con respecto a la microestructura, todos los lemas o cabeza de los artículos están seguidos por su pronunciación encerrada entre barras, paréntesis o corchetes. Algunos diccionarios emplean los símbolos del Alfabeto Fonético Internacional, y la mayoría explica en las hojas preliminares cómo podría pronunciarse cada símbolo fonético. Seguido de la pronunciación se localiza la categoría gramatical de la palabra (sustantivos, adjetivos...) de manera abreviada y sus respectivos equivalentes en la lengua materna. Si la palabra tiene varias formas gramaticales, el diccionario lo indica. Algunos presentan las formas verbales, (verbos transitivo o intransitivo), las formas del pasado y participio si el verbo es irregular, y su empleo como verbo frasal.

b) Los distintos equivalentes o acepciones están marcados por medio de números, guiones o doble barra. Algunos diccionarios presentan las voces estrechamente relacionadas con la principal dentro de la misma entrada, otros los desarrollan en un artículo diferente (con una entrada aparte). La mayoría presenta los plurales de sustantivos irregulares, mas muy pocos muestran cuando el sustantivo es incontable y cómo se forma el comparativo y superlativo de los adjetivos cortos. 
c) Igualmente, la mayoría de los diccionarios bilingües inglés-español/español-inglés, aportan ejemplos, frases lexicales, modismos e indicaciones entre paréntesis que ayudan al aprendiz a seleccionar el equivalente más indicado según el contexto. No obstante, muchas veces las indicaciones entre paréntesis no están escritas en la lengua materna, lo que las hacen poco útiles para el alumno.

d) En cuanto a los artículos se muestra una serie de elementos gráficos, (símbolos y abreviaturas de uso estilísticos: coloquial, popular, regional, científico...) diseñados para facilitar su uso, que a su vez lo hacen complejo.

e) Los elementos micro estructurales observados también tienen correlación con los criterios de Pérez, (ob.cit) sobre la microestructura; aunque no todos los diccionarios los poseen todos. Los únicos diccionarios más completos según los fundamentos lexicográficos del mencionado autor, son el Oxford Pocket y el Larousse School.

\section{El Vocabulario y la Categoría Gramatical}

Los planteamientos presentados por Harmer (2001) sobre el vocabulario y la categoría gramatical de la palabra muestran algunas de las dificultades que enfrentan los estudiantes al momento de buscar equivalencias léxicas en diccionarios bilingües inglésespañol.

\section{Word Meaning}

En inglés una misma palabra puede tener diferentes significados, lo que este autor llama "polisemia" y lo presenta en el siguiente ejemplo: When you have read your book you can ring up a restaurant and book a table, but if you drive too fast on the way you might be booked for speeding. Al respecto, Harmer expresa que "this polysemy is only resolved when we see the word in context". Es decir, el significado de una palabra va a depender del contexto en el que se encuentre.

En estos casos, algunos diccionarios como el Oxford, Norma y Larousse entre otros, presentan ejemplos de palabras con esas características ubicadas en contexto para indicar al usuario el equivalente más adecuado. Es por ello que el docente debe procurar que el aprendiz maneje dicha clase de información. El siguiente es un ejemplo tomado del Oxford (2006) (Fig. 1). 


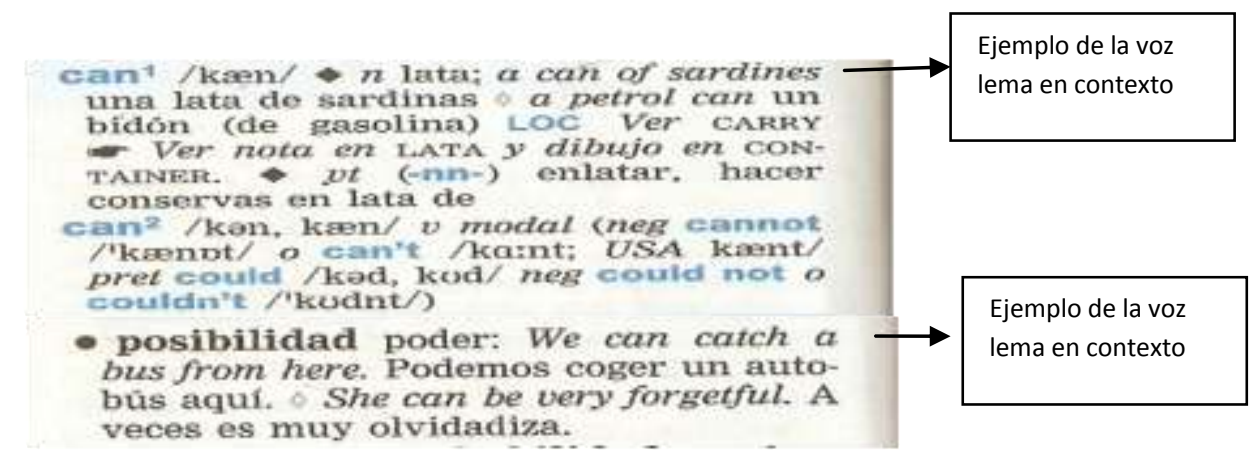

Figura 1. Ejemplo de la voz lema en contexto

Como se puede observar, la palabra can según el contexto en el cual se emplee, puede significar "lata, o poder (posibilidad)" y el mismo diccionario presenta ejemplos para su discriminación.

\section{Combinación de Palabras}

Según Harmer (ob.cit), "Although words can appear as single items which are combined in a sentence, they can also occur in two-or-more item groups". Es decir, una palabra puede tener un significado en determinada oración, pero según su combinación con otras palabras, dicha combinación puede tener un significado diferente al significado de cada palabra vista de manera aislada. En este sentido, el grupo de palabras debe percibirse y traducirse como una unidad. Las combinaciones de palabras se clasifican, según este autor, en las siguientes:

Verbos frasales: según Harmer (ob.cit), "these are formed by adding an adverb or a preposition to a verb to create new meanings". El significado de estos verbos va a depender de la partícula que le siga, por ello no se deben traducir de manera aislada, sino como una unidad. Ejemplo: "set out" (emprender un viaje), We set out tomorrow (saldremos mañana). En este ejemplo set out tiene un significado totalmente diferente a set visto de forma aislada, como en set the table (poner la mesa). La mayoría de los diccionarios bilingües estudiados presentan algunos verbos frasales; muestran su traducción y proporcionan ejemplos.

Collocations: las colocaciones consisten en la manera en que las palabras son combinadas con otras palabras. Existen combinaciones que se pueden hacer y otras que no. Ejemplo: se puede decir "do your homework" (haz tu tarea), y esta es una colocación aceptable, pero, "make your homework" no lo es, debido a que make semánticamente 
tiende a significar construir, crear o fabricar, y el verbo do se relaciona con la realización de actividades. Algunos diccionarios como el Oxford presentan ejemplos de Collocations. Por ello se insiste en que el estudiante debe aprender a usar el diccionario correctamente.

Palabras compuestas: son aquellas palabras que están formadas por dos morfemas libres, tales como: "traffic light, workshop, notebook."

Frases lexicales: "lexical phrases are like prefabricated building units", Harmer (ob.cit). Entre las frases lexicales, según Baigent, citada por Harmer (ob.cit), se encuentran las frases funcionales (Ej. by the way, on the other hand), idiomáticas (Ej. an only child) y expresiones verbales (Ej. can't afford to, don't mind).

De todos estos aspectos planteados por Harmer (ob.cit) hasta el momento, los que presentan una mayor dificultad para su aprendizaje son las frases lexicales y las colocaciones, ya que sobre éstos no existe regla para su enseñanza. Estos planteamientos teóricos resultan relevantes para el presente estudio al mostrar indirectamente las dificultades que enfrentan los estudiantes al momento de buscar equivalencias léxicas en diccionarios bilingües inglés-español; a su vez, suministra fundamentos que deben ser tomados en cuenta en la realización de las actividades prácticas del manual propuesto.

\section{Categoría Gramatical de las Palabras (Cambios Morfológicos)}

Una palabra puede cambiar morfológicamente y éste a su vez puede afectar su categoría gramatical y su significado. Estos cambios morfológicos se deben a los múltiples afijos que se les pueden adherir a los vocablos. Según Harmer (ob.cit), hay afijos que no cambian el sentido de las palabras ni su categoría gramatical como el morfema <s> para la formación del plural y la conjugación de los verbos en tercera persona. Entre los morfemas que cambian el significado de las palabras el autor en cuestión presenta los siguientes: <im y dis>, Ejemplo: Possible and impossible, agree and disagree.

Con respecto a la forma gramatical, dicho autor expresa que "words can also occupy more than one word class, a fact that is frequently (but not always) indicated by morphological change". A continuación se presenta en el cuadro 1 manera en que las palabras pueden ocupar diferentes partes del habla, algunas con alteraciones morfológicas y otras sin alteraciones. 


\section{Cuadro 1: Partes del habla que pueden ocupan una misma palabra}

\begin{tabular}{|lllc|}
\hline Verb & noun & adjective & adverb \\
Argue & argument & argumentative & argumentatively \\
Anger & anger & angry & angrily \\
Suggest & suggestion & suggestive & suggestively \\
Calm & calm & calm & calm \\
\hline
\end{tabular}

Lo que se consideró de este último planteamiento para este estudio, es lo concerniente a los cambios morfológicos. Después de la revisión detallada de algunos diccionarios inglés-español/español-inglés, se observó que en el corpus no presentan los verbos conjugados en tercera persona, el pasado de los verbos regulares, ni el plural de sustantivos regulares. No obstante, algunos exhiben artículos sobre el pasado de verbos irregulares sin mencionar su traducción; sólo indican al usuario que dicho verbo es el pasado de un verbo en particular. Igualmente proporcionan el plural de sustantivos irregulares.

Además, se apreció que existen palabras que sufren cambios morfológicos (formas adverbiales con el sufijo $<$ ly $>$ y las formas comparativas y superlativas de adjetivos cortos con los sufijos <er> y <est>) que no aparecen en algunos de los diccionarios. Todos estos aspectos y los planteamientos expuestos por Harmer (ob.cit) son el fundamento principal sobre el cual se basan las actividades de uso del diccionario del manual.

\section{Fundamentos Teóricos para la Elaboración de Manuales Didácticos del Instituto Técnico de Capacitación y Productividad (INTECAP) (2008).}

Finalmente, y para complementar los pilares en los cuales se basa el objetivo del presente estudio, se consideró los fundamentos teóricos para la elaboración de manuales didácticos del Instituto Técnico de Capacitación y Productividad (INTECAP). Allí se describen los elementos que se deben tomar en cuenta en la elaboración de manuales didácticos.

A continuación se presenta la definición de algunos términos que deben tenerse claros al momento de diseñar manuales didácticos según INTECAP: Dichas definiciones son tomadas de los lineamientos expuestos por INTECAP en sus escritos sobre el tema en cuestión. 
Didáctica: es una disciplina orientada en mayor grado hacia la práctica, cuyo objetivo primordial es orientar la enseñanza. La enseñanza por su parte, no es más que la dirección del aprendizaje. La didáctica a su vez está constituida por un conjunto de procedimientos y normas destinados a dirigir el aprendizaje de la manera más eficiente que sea posible.

Pedagogía: es la ciencia que se ocupa de la educación y la enseñanza. Esta ciencia proporciona guías para planificar, ejecutar y evaluar procesos de enseñanza y aprendizaje. En griego, paida o paidos significa niños, en oposición a andro que indica hombre. En base a esto se distingue entre Pedagogía (enseñar a niños) y Andragogía (enseñar a adultos).

Mediación pedagógica: es un proceso para lograr una comunicación efectiva en la transmisión de contenidos educativos, entre diseñadores y participantes, a través de materiales didácticos.

Diseño gráfico: es el producto de un proceso creativo de comunicación que estéticamente armoniza figuras, textos, colores, formas y otros elementos icónicos para plasmarlos en un espacio real o virtual.

Diagramación de manuales didácticos: son los procesos que el diseñador gráfico realiza para ordenar textos, gráficas, esquemas, fotografías y otros elementos gráficos dentro de una página, con el objetivo de comunicar visual y estéticamente los contenidos en forma didáctica, acorde a los niveles de percepción del estudiante.

En el INTECAP se reconocen dos tipos de manuales didácticos: manuales técnicos y material de apoyo didáctico. Para el diseño del manual propuesto se consideró este último por su simplicidad en el diseño y la didáctica.

Material de apoyo didáctico (MAD): Es un documento de comunicación didáctica estructurado, que a través de textos específicos y elementos icónicos (ilustraciones, gráficas, etc.), se utiliza en el proceso de enseñanza aprendizaje para contribuir a que el participante desarrolle competencias (habilidades, destrezas, conocimientos y actitudes) como apoyo para la formación.

\section{Informaciones de carácter técnico:}

El contenido debe ser adaptable al entorno tecnológico y suficiente para lograr la competencia del participante, con ilustraciones y diagramas de apoyo para la asimilación de las técnicas de trabajo, con contenidos tecnológicamente actualizados. 


\section{Utilidad de los materiales:}

Los productos deben ser utilizados por los participantes, presentando únicamente la información que es de utilidad, evitando informaciones de relleno o innecesarias. Los contenidos deben entenderse claramente, ser veraces y actuales.

\section{Presentación y diagramación:}

Nitidez y claridad del producto, ordenamiento visual del diseño, orden lógico de la presentación de la información, uso suficiente de información gráfica y analítica, etc.

\section{Pertinencia entre la necesidad del producto y el producto:}

El producto debe responder a una necesidad y ser útil para el trabajo.

\section{Enfoque pedagógico:}

Corresponde a un orden, desglose de unidades o temas, equilibrio informativo y ejercicios. Debe estar diseñado para las características de la población meta, nivel de escolaridad, capacidades psicomotrices y psicomotoras, disponibilidad del tiempo etc., con estrategias de formación adecuadas al participante y leguaje claro. Para facilitar la enseñanza es necesario adjuntar o realizar gráficas, fotografías, dibujos y demás elementos que permitan completar su trabajo garantizando que las mismas sean claras y con definición correcta.

\section{METODOLOGÍA}

El presente trabajo está enmarcado dentro de la modalidad de Proyecto Especial, ya que de acuerdo con lo estipulado en el Manual de Trabajos de Grado, Especialización y Maestría y Tesis Doctorales de la UPEL (2006), se corresponden a esta modalidad los "Trabajos que lleven a creaciones tangibles, susceptibles de ser utilizadas como soluciones a problemas demostrados... se incluyen en esta categoría los trabajos de elaboración de libros de textos y de materiales de apoyo educativos". En este sentido, la presente investigación busca la elaboración de un manual para el uso correcto del diccionario bilingüe inglés-español, a través del cual se espera transformar la realidad evidenciada en los estudiantes que conforman el campo de estudio.

Por otra parte, se apoya en un estudio de campo, el cual consiste en "el análisis sistemático de problemas en la realidad, con el propósito bien sea de describirlos, explicar sus causas y efectos, o predecir su ocurrencia... (y) los datos de interés son recogidos de 
forma directa de la realidad", Manual de la UPEL, (ob.cit). Tal como se ha citado, los datos de este estudio fueros recogidos de fuentes primarias; a partir de los cuales se realizó el diagnóstico que dio origen a la creación del manual antes descrito. Es importante señalar que esta investigación de campo es de carácter descriptivo debido a que pretende describir la realidad existente para tomar decisiones posteriores a partir de la misma.

\section{Sujetos de estudio}

La población en este estudio está conformada por 40 estudiantes correspondientes a dos (2) secciones de cuarto (4to) año, de la U.E. "Rafael Antonio Mujica" del año escolar (2010-2011). En virtud de que la población es pequeña, no se seleccionó muestra y se tomó la totalidad para la indagación. Según Muñoz (2005), “es válido cuando las poblaciones son reducidas, pasando todos sus integrantes a ser sujetos de estudio" razón por la cual a la población de este trabajo se le llama sujetos de investigación.

\section{Procedimiento}

Esta investigación se desarrolló en tres fases. En la primera se realizó el diagnóstico de necesidades, en la segunda se elaboró la propuesta (manual) como respuesta a la necesidad evidenciada y en la tercera se validó.

\section{Fase I: Diagnóstico de las Necesidades.}

En esta fase se determinó la situación objeto de estudio con la finalidad de conocer la necesidad de los estudiantes de cuarto año de la U.E. "Rafael Antonio Mujica" en cuanto a la elaboración de un manual para el aprendizaje del uso del diccionario bilingüe inglésespañol. A su vez se recabó información bibliográfica relacionada con el tema de estudio y se indagó sobre trabajos similares con el objeto de orientar esta investigación a través de exploraciones precedentes y hallazgos encontrados por otros investigadores.

Para lograr esta primera etapa se elaboró un instrumento para recolectar la información necesaria procedente de la población objeto de estudio. El instrumento consistió en un test (prueba) cognitivo, con la designación dada por Aiken (1996), quien usa la expresión test de habilidades para designar todos aquellos test cuya función es determinar conocimiento.

Previamente a dicho instrumento se le determinó la validez a través de juicios de expertos. Igualmente se calculó la confiabilidad en la cual se determinó el índice de 
discriminación de los ítemes $(0,66$ = nivel de discriminación alto) e índice de dificultad (50 = nivel de dificultad moderado). Posteriormente, se calculó la consistencia interna del instrumento a través del método Kuder-Richardson en la cual se obtuvo una confiabilidad de 0,77 , lo cual corroboró que el instrumento podía ser aplicado puesto que tenía un nivel de confiabilidad alto. Una vez aplicado el instrumento a la población en estudio los resultados fueron analizados a través de cuadros y gráficos de los cuales se desprendieron las conclusiones y recomendaciones.

\section{Análisis de los Datos}

La información requerida en el estudio se obtuvo mediante la aplicación de la prueba de habilidad que fue respondida por los participantes haciendo uso de sus diccionarios. De esta forma se pudo conocer los conocimientos de los estudiantes en cuanto al uso del mencionado instrumento.

Una vez obtenido los datos se llevó a cabo el análisis e interpretación cumpliendo de esta forma con el objetivo referente al diagnóstico de necesidad. La codificación consistió en asignarle un número a cada respuesta (El $\mathrm{N}^{\circ}$ uno 1 para las respuestas correctas y 0 para las incorrectas). En la primera parte del instrumento constituida por 10 ítemes de respuestas dicotómicas (sí o no) se sumaron las respuestas correctas y se tabularon a través de porcentajes que luego se presentaron por medio de gráficos de barras.

En la segunda parte constituida por 4 ítemes se le dio un tratamiento diferente considerando que cada ítem estaba conformado por 4 ejercicios de completación. En este sentido, una vez analizado los datos fueron categorizados para ser presentados a través de gráficos de barras. La categorización consistió en asignarle un número del 1 al 4 según los ejercicios acertados. De esta manera se asignó el 4 (excelente) si respondían correctamente los cuatro ejercicios propuestos por ítem, 3 (bueno) si acertaban tres, 2 (regular) si acertaban dos, 1 (deficiente) si acertaban 1 y 0 (muy deficiente) si no acertaban ninguno de los ejercicios planteados. Este análisis fue realizado de forma manual por la autora de esta investigación.

\section{DESDE LA LUZ DE LOS RESULTADOS}

En los instrumentos de recolección de información se obtuvieron los siguientes datos por subcategoría. En la subdimensión: instrucciones de uso se obtuvo un 55\% de respuestas erradas, en la subdimensión: lista de abreviaturas un 45\%, apéndices, 25\%, 
$90 \%, 37,5 \%$, uso adecuado de algunos elementos que conforman la macroestructura $85 \%$, y en la subdimensión uso adecuado de algunos elementos incorporados a la microestructura $12,5 \%$ y $52,5 \%$ de respuestas incorrectas.

En lo referente a los ítemes sobre la búsqueda de equivalencias léxicas en el diccionario, en la subdimensión palabras compuestas solo 1 (2,5\%) estudiante respondió los 4 ejercicios correctamente. En la subdimensión: palabras con cambios morfológicos ningún estudiante $(0 \%)$ respondió los cuatro ejercicios satisfactoriamente. En la subdimensión: word meaning $8(20 \%)$ alumnos de los 40 participantes contestaros correctamente los cuatro ejercicios propuestos en el ítem, y en la subdimensión: combinación de palabras, sólo $1 \quad(2,5)$ estudiante respondió los cuatro ejercicios correctamente.

A través de los resultados obtenidos, tanto en la primera parte del instrumento y más afianzada en la segunda parte, justifican la propuesta del diseño del manual para el uso del diccionario bilingüe inglés-español/español-inglés dirigido a los estudiantes de cuarto año de la U. E. "Rafael Antonio Mujica", ya que se pudo evidenciar las deficiencias que presentan los participantes en cuanto al empleo adecuado del mencionado instrumento. Es importante destacar que la mayoría de los educandos presentaron más dificultad en la búsqueda de equivalentes de palabras con cambios morfológicos (verbos conjugados en tercera persona, en pasado simple y sustantivos en plural) y frases lexicales. En lo referente a los cambios morfológicos ningún estudiante $(0 \%)$ respondió los cuatro ejercicios propuestos en el ítem y sólo 2 (5\%) respondieron favorablemente 3 de los cuatro ejercicios. En cuanto a las frases lexicales 31 estudiantes equivalentes al 77,5\% no acertaron ninguno de los ejercicios propuestos en el ítem. Tal como lo indican los resultados se demuestra la necesidad de crear una herramienta o buscar una solución para ayudar a los estudiantes a hacer uso adecuado del diccionario bilingüe Inglés-español; español-inglés.

\section{CONCLUSIONES}

El diccionario bilingüe Inglés-Español es una herramienta valiosa en el aprendizaje del inglés como lengua extranjera. Sin embargo, esta herramienta podría entorpecer dicho aprendizaje si no es empleada adecuadamente. Para el correcto uso del diccionario bilingüe inglés-español, es necesario que el aprendiz conozca cómo se estructura. Éste consta de 
una serie de apartados y elementos gráficos (símbolos, abreviaturas, indicaciones, ejemplos) diseñados para facilitar su uso. No obstante, no todos los usuarios conocen de ello, o bien, no saben en que les puede ayudar. A través de este estudio se pudo corroborar algunas de las dificultades que presentan los aprendices de cuarto año de la U.E. "Rafael Antonio Mujica" al momento de buscar equivalencias léxicas en los diccionarios.

En cuanto a la macro estructura de los diccionarios bilingües inglés- español, los participantes objeto de estudio presentaron pocas dificultades pero relevantes para la investigación, ya que se pudo evidenciar que aunque un gran porcentaje está consiente que sus diccionarios poseen listas de verbos y listas de números, la gran mayoría desconoce la presencia de los apéndices referentes a la información gramatical. Asimismo se pudo conocer que muchos están al tanto que sus diccionarios tienen instrucciones de uso y lista de abreviaturas, pero en la realización de los ejercicios se evidenció que a pesar de ello presentan dificultades significativas en la búsqueda de equivalencias léxicas; además un gran número de estudiantes manifestó desconocer el uso de las palabras guías lo cual es una justificación más para el diseño del manual.

Por otra parte, los argumentos que sustentan con mayor ahínco la presente propuesta (diseño de un manual didáctico para el uso del diccionario bilingüe inglésespañol), tienen relación con las dificultades que presentaron los estudiantes al buscar equivalencias léxicas en los diccionarios. Aunque se evidenciaron dificultades generales, las dificultades más relevantes se observaron en la búsqueda de equivalentes de palabras con cambios morfológicos como verbos conjugados en tercera persona, verbos en pasado y sustantivos en plural. Igualmente se observaron muchas dificultades en la búsqueda de equivalencias de frases lexicales. Por tales razones se concluye que:

1. Al momento de enseñar como usar el diccionario bilingüe es conveniente explicar a los alumnos que el significado de una palabra no se debe tomar de manera arbitraria. Es decir, se debe considerar el contexto en el cual se encuentre el término y su combinación con otras palabras. Asimismo, es necesario dar a conocer a los estudiantes que muchos diccionarios como el Oxford, Larousse y el Norma, entre otros, presentan ejemplos para ubicar mejor al usuario.

2. Es necesario que los aprendices revisen la información adicional que se encuentra en dicho recurso y que estén conscientes de su utilidad para que puedan emplearla 
en el momento oportuno. De nada sirve que conozcan que el diccionario muestra una lista de abreviaturas si no se les informa en qué le puede servir, igualmente sucede con las listas de verbos, la información gramatical y fonética.

3. Es preciso recordar a los estudiantes que las palabras están organizadas alfabéticamente y darles a conocer que al momento de buscar un vocablo no sólo deben fijarse en la primera letra, sino también en las subsiguientes, siempre en orden alfabético. Es menester además mostrarles la utilidad de las palabras guías para que la búsqueda se haga más sencilla.

4. Por otra parte, es común encontrarse con estudiantes que expresan no conseguir la palabra que buscan, y la causa se debe muchas veces a que lo hacen en el segmento equivocado. Por ello, el docente debe hacer énfasis en el uso de cada segmento. El de inglés-español se emplea para buscar el equivalente en español de palabras escritas en inglés y el de español-inglés sirve para buscar el equivalente en inglés de palabras escritas en español. Es elemental que los aprendices se identifiquen con su diccionario para que no tengan dificultades de esta índole.

5. Es importante que también conozcan la utilidad de la información gramatical que ofrecen los diccionarios, en especial las relacionadas con las partes del habla o categoría gramatical de las palabras (Verbos, adjetivos, entre otros) para que puedan identificarlas correctamente cuando estén trabajando con la microestructura o estructura de los artículos. También es conveniente que tengan conocimiento sobre el empleo del apartado sobre la pronunciación. Ya que de nada les sirve conocer que sus diccionarios proporcionan la pronunciación de las palabras si no la saben usar. Todos estos hallazgos son los que suministraron las directrices que orientaron la elaboración del manual propuesto.

\section{Recomendaciones}

A partir de los hallazgos encontrados a través de la presente investigación se recomienda que los resultados sean dados a conocer a los docentes de inglés que laboran en la U.E. "Rafael Antonio Mujica" con la Tercera Etapa de Educación Básica para que consideren las conclusiones de este trabajo e incluyan en su programación el uso adecuado del diccionario, inclusive desde el primer año. 
El manual sobre el uso del diccionario bilingüe debe ser aplicado a los estudiantes que conformaron el campo de estudio para validar su efectividad en cuanto al tema.

Esta investigación debe ser difundida a otras instituciones educativas para que los beneficios que se puedan obtener de la misma no se limiten a una minoría de estudiantes sino que pueda ser aplicado a otras poblaciones estudiantiles que presenten las mismas características de los sujetos de estudio.

La enseñanza del uso adecuado del diccionario bilingüe inglés-español debe ser considerada desde el primer año, por lo que es conveniente que los docentes de inglés tomen al menos una clase en el año escolar para trabajar con el uso del diccionario.

Considerando que algunas instituciones universitarias, en las clases de inglés que se imparte en las diferentes carreras que ofertan, contemplan una clase para abordar el tema del uso adecuado del diccionario, en la nueva propuesta curricular del Ministerio del Poder Popular para la Educación debería considerarse al menos una clase para la enseñanza del uso adecuado del diccionario bilingüe inglés-español en los programas de inglés de Tercera Etapa de Básica y Diversificado.

\section{REFERENCIAS}

Aiken, L. (1996). Test Psicológicos y Evaluación, México: Prentice Hall.

Bajo Pérez, E. (2000). Diccionarios: Introducción a la historia de la lexicografía del español. España: Trea.

Benedikt A., M. (2005). A la hora de comprar un diccionario bilingüe [Documento en línea]. Universidad de Salamanca. Disponible: <http://www.g-dafes.net/chancen/hilfsmittel/diccionario.pdf>. [Consulta: 2010, Febrero 22]

Cote González, M. y Tejedor Martínez, C. (1998). La enseñanza de vocabulario y el uso del diccionario. Encuentro. Revista de Investigación e Innovación en la Clase de Idiomas [Revista en línea], 10 pp. 26-35. Disponible: http://www.encuentrojournal.org/textos/La_Ense_anza_de_Vocabulario_y_el_Uso_de 1_Diccionario.pdf [Consulta: 2011, Agosto 16]

Distribuidora Escolar. (2009). University of Miami dictionary: English-Spanish, Spanish-English: The language spoken today. Guarenas, Venezuela: Autor.

Enecoiz Osinaga, M., I. (2000). Utilidad del diccionario para la traducción de textos en las clases de ele [Documento en línea]. Ponencia presentada en el XI Congreso Internacional de ASELE, (pp. 287-296). Disponible: 
http://cvc.cervantes.es/ensenanza/biblioteca_ele/asele/pdf/11/11_0287.pdf [Consulta: 2011, julio 22]

Fernández de Bobadilla Lara, N. (1999). Hacia un uso correcto del diccionario en la lectura de textos científicos en inglés. Encuentro. Revista de Investigación e Innovación en la clase de idiomas [Revista en línea], 11, 96-105. Disponible: http://www.encuentrojournal.org/textos/11.11.pdf [Consulta: 2010, Mayo 20]

Goldsmith, P., Pérez, A. y Ashby, M. (1996). Oxford pocket: Para estudiantes de inglés: español-inglés, inglés -español. México: Oxford University Press.

Harmer, J. (2001). The practice of English language teaching (3a. ed.). Malaysia: Longman.

Instituto Técnico de Capacitación y Productividad. (2008). Elaboración de manuales didácticos [Libro en línea]. (2 $\mathrm{a}^{\mathrm{a}}$ ed.). Guatemala. Disponible: http://intecap.info/public/manuales/divisiontecnica/ISDT11\%20\%20\%20E2.pdf [Consulta: 2011, Julio 14]

Kingkolor. (2008). Dictionary Tower Chicago Illustrated: English -Spanish, SpanishEnglish. Bogotá, Colombia: Autor.

Larousse. (2002). Larousse diccionario básico: Español-inglés/ inglés-español (4a.ed.). México: Autor.

Larousse. (2007). Larousse diccionario pocket español inglés/inglés español. México: Autor.

Larousse. (2005). El pequeño Larousse ilustrado (11a. ed.). México: Autor.

Llorente, L., I. y Fitzhugh Parra, M. (s.f). Uso del diccionario en la clase de traducción: Algunas reflexiones. RedELE Revista Electrónica de Didáctica / Español Lengua Extranjera [Revista en línea], 16, 1571-4667. Disponible: http://www.aulaintercultural.org/IMG/pdf/Llorente_Parra-Diccionario.pdf [Consulta: 2011, Julio 30]

Luppescu, S. y Day, R. (1993). Reading dictionaries and vocabulary learning: Language Learning, 43(2), 263-287.

Mundo Cultural. (2004). Dictionary Chicago Universal. Spanish- English, English Spanish. México: Autor.

Muñoz, E. (2005). Cómo elaborar una tesis de grado. Méjico: Trillas.

Norma. (2003). Diccionario bilingüe básico: Español-inglés, inglés -español. Colombia: Autor. 
Pérez, F. J. (2005). Pensar y hacer el diccionario. Caracas: Los Libros de El Nacional.

Rapsa, (2002) New dictionary Chicago city: Español- inglés, inglés-español. Colombia: Autor.

Universidad Pedagógica Experimental Libertador. (2006). Manual de trabajos de grado, especialización y maestría y tesis doctorales (4a. ed.). Caracas: FEDUPEL.

Zúñiga Espitia, G. (2010). New grand Chicago universal dictionary: Inglés-español/ español-inglés. Bogotá, Colombia: Nika 